\title{
NONEXISTENCE OF WEAKLY ALMOST COMPLEX STRUCTURES ON GRASSMANNIANS
}

\author{
TANG ZI-ZHOU
}

\author{
(Communicated by Thomas Goodwillie)
}

\begin{abstract}
In this paper we prove that, for $2 \leq k \leq n / 2$, the unoriented Grassmann manifold $G_{k}\left(\mathbb{R}^{n}\right)$ admits a weakly almost complex structure if and only if $n=2 k=4$ or 6 ; for $3 \leq k \leq \frac{n}{2}$, none of the oriented Grassmann manifolds $\widetilde{G}_{k}\left(\mathbb{R}^{n}\right)$-except $\widetilde{G}_{3}\left(\mathbb{R}^{6}\right)$ and a few as yet undecided ones-admits a weakly almost complex structure.
\end{abstract}

\section{INTRODUCTION}

For $1 \leq k<n$, let $\widetilde{G}_{k}\left(\mathbb{R}^{n}\right) \quad\left(G_{k}\left(\mathbb{R}^{n}\right)\right.$ resp.) denote the oriented (unoriented) Grassmann manifold of the oriented (unoriented) $k$-dimensional vector subspace of $\mathbb{R}^{n}$. $\widetilde{G}_{k}\left(\mathbb{R}^{n}\right) \quad\left(G_{k}\left(\mathbb{R}^{n}\right)\right)$ is a smooth manifold of dimension $k(n-k)$. Note that $\widetilde{G}_{1}\left(\mathbb{R}^{n}\right) \cong S^{n-1}\left(G_{1}\left(\mathbb{R}^{n}\right) \cong R P^{n-1}\right)$, the $(n-1)$-sphere (real projective space), and that $\widetilde{G}_{k}\left(\mathbb{R}^{n}\right) \cong G_{n-k}\left(\mathbb{R}^{n}\right) \quad\left(G_{k}\left(\mathbb{R}^{n}\right) \cong G_{n-k}\left(\mathbb{R}^{n}\right)\right)$ under the diffeomorphism that sends a $k$-plane $V$ to its orthogonal complement $V^{\perp}$.

Recall that a smooth manifold $M$ is said to be (weakly) almost complex if its tangent bundle $\tau M$ is (stably) isomorphic to the realification of a complex vector bundle over $M$.

For example, $\widetilde{G}_{1}\left(\mathbb{R}^{n}\right) \cong S^{n-1}$ is weakly almost complex for all $n$ but is almost complex only when $n=3$ or $7[1] ; G_{1}\left(\mathbb{R}^{n}\right) \cong R P^{n-1}$ is weakly almost complex only when $n$ is even. It is a classical result that $\widetilde{G}_{2}\left(\mathbb{R}^{n}\right) \cong$ $\mathrm{SO}(n) /(\mathrm{SO}(2) \times \mathrm{SO}(n-2))$ is a Hermitian symmetric space and is therefore almost complex for all $n$. Our main results are:

Theorem 1.1. Let $2 \leq k \leq \frac{n}{2}$. Then $G_{k}\left(\mathbb{R}^{n}\right)$ is weakly almost complex if and only if $n=2 k=4$ or 6 .

Theorem 1.2. Let $3 \leq k \leq \frac{n}{2}$. Then $\widetilde{G}_{k}\left(\mathbb{R}^{n}\right)$ is not weakly almost complex if $n$ is odd or $(n-k) \geq 8$.

Our results are sharper than that in [6]. Note that $\widetilde{G}_{3}\left(\mathbb{R}^{6}\right)$ is weakly almost complex [6]. The unsolved cases for weak complexity of $\widetilde{G}_{k}\left(\mathbb{R}^{n}\right)$ are $\widetilde{G}_{4}\left(\mathbb{R}^{8}\right)$, $\widetilde{G}_{5}\left(\mathbb{R}^{10}\right), \quad \widetilde{G}_{6}\left(\mathbb{R}^{12}\right), \quad \widetilde{G}_{7}\left(\mathbb{R}^{14}\right), \quad \widetilde{G}_{3}\left(\mathbb{R}^{8}\right), \quad \widetilde{G}_{4}\left(\mathbb{R}^{10}\right), \quad \widetilde{G}_{5}\left(\mathbb{R}^{12}\right)$, and $\widetilde{G}_{3}\left(\mathbb{R}^{10}\right)$. Let

Received by the editors November 4, 1992.

1991 Mathematics Subject Classification. Primary 57R15. 
$\widetilde{\gamma_{n, k}}\left(\gamma_{n, k}\right)$ denote the canonical $k$-plane bundle over $\widetilde{G}_{k}\left(\mathbb{R}^{n}\right) \quad\left(G_{k}\left(\mathbb{R}^{n}\right)\right)$, and let $\widetilde{\beta}_{n, k}\left(\beta_{n, k}\right)$ be its orthogonal complement, whose fiber over a $V \in \widetilde{G}_{k}\left(\mathbb{R}^{n}\right)$ $\left(G_{k}\left(\mathbb{R}^{n}\right)\right)$ is the vector space $V^{\perp} \subset \mathbb{R}^{n}$. We have bundle equivalence

$$
\widetilde{\gamma_{n, k}} \oplus \widetilde{\beta_{n, k}} \cong n \varepsilon \quad\left(\gamma_{n, k} \oplus \beta_{n, k} \cong n \varepsilon\right),
$$

where $\varepsilon$ denotes a trivial line bundle.

It is well known that the tangent bundle $\tau \widetilde{G}_{k}\left(\mathbb{R}^{n}\right)\left(\tau G_{k}\left(\mathbb{R}_{n}\right)\right)$ of $\left.\widetilde{G}_{k}\left(\mathbb{R}^{n}\right)\right)$ has the following description (see [4]):

$$
\tau \widetilde{G}_{k}\left(\mathbb{R}^{n}\right) \cong \widetilde{\gamma_{n, k}} \otimes \widetilde{\beta_{n, k}} \quad\left(\tau G_{k}\left(\mathbb{R}^{n}\right) \cong \gamma_{n, k} \otimes \beta_{n, k}\right) .
$$

Using (1.3) and (1.4), we obtain

(1.5) $\left.\tau \widetilde{G}_{k}\left(\mathbb{R}^{n}\right) \oplus \widetilde{\gamma_{n, k}} \otimes \widetilde{\gamma_{n, k}}\right) \cong n \widetilde{\gamma_{n, k}} \quad\left(\tau G_{k}\left(\mathbb{R}^{n}\right) \oplus\left(\gamma_{n, k} \otimes \gamma_{n, k}\right) \cong n \gamma_{n, k}\right)$.

For a CW complex $X$, let $r: K(X) \rightarrow K O(X)$ denote the homomorphism of Abelian groups gotten by restriction of scalars to $\mathbb{R}$, and let $c: K O(X) \rightarrow K(X)$ denote the complexification, $c[\xi]=\left[\xi \otimes_{\mathbb{R}} \mathbb{C}\right]$, which is a ring homomorphism.

We have the following identity:

$$
r c(x)=2 x \quad \forall x \in K O(X) .
$$

\section{THE UNORIENTED GRAsSMANNIANS}

Lemma 2.1. $G_{2}\left(\mathbb{R}^{6}\right)$ is not weakly almost complex.

Proof. It is well known that

$$
H^{*}\left(G_{2}\left(\mathbb{R}^{6}\right) ; \mathbb{Z}_{2}\right) \cong \mathbb{Z}_{2}\left[w_{1}, w_{2}, \bar{w}_{1}, \bar{w}_{2}, \bar{w}_{3}, \bar{w}_{4}\right]
$$

modulo the relation $\left(1+w_{1}+w_{2}\right)\left(1+\bar{w}_{1}+\bar{w}_{2}+\bar{w}_{3}+\bar{w}_{4}\right)=1$, so

$$
H^{*}\left(G_{2}\left(\mathbb{R}^{6}\right) ; \mathbb{Z}_{2}\right) \cong \mathbb{Z}_{2}\left[w_{1}, w_{2}\right] /\left\langle w_{1}^{5}+w_{1} w_{2}^{2}, w_{1}^{2} w_{2}^{2}+w_{1}^{4} w_{2}+w_{2}^{3}\right\rangle .
$$

The fact $H^{8}\left(G_{2}\left(\mathbb{R}^{6}\right) ; \mathbb{Z}_{2}\right) \cong \mathbb{Z}_{2}$ implies $w_{2}^{4} \neq 0$. By (1.5), the total StiefelWhitney classes of $G_{2}\left(\mathbb{R}^{6}\right)$ are given by

$$
w\left(G_{2}\left(\mathbb{R}^{6}\right)\right)=\left(1+w_{1}+w_{2}\right)^{6} /\left(1+w_{1}^{2}\right)=1+\left(w_{1}^{4}+w_{2}^{2}\right)+w_{1}^{2} w_{2}^{2}+w_{2}^{4} .
$$

This gives

$$
w_{2}\left(G_{2}\left(\mathbb{R}^{6}\right)\right)=0, \quad w_{8}\left(G_{2}\left(\mathbb{R}^{6}\right)\right)=w_{2}^{4} \neq 0 .
$$
[5]:

The following results follow immediately from Wu's formula $s q^{1} w_{2}=w_{1} w_{2}$

$$
\begin{aligned}
& s q\left(w_{1}^{6}\right)=w_{1}^{6}, \quad s q\left(w_{1}^{4} w_{2}\right)=w_{1}^{4} w_{2}+w_{1}^{5} w_{2}, \\
& s q\left(w_{1}^{2} w_{2}^{2}\right)=w_{1}^{2} w_{2}^{2}, \quad s q\left(w_{2}^{3}\right)=w_{2}^{3}+w_{2}^{3} w_{1} .
\end{aligned}
$$

Therefore, $s q^{2}: H^{6}\left(G_{2}\left(\mathbb{R}^{6}\right) ; \mathbb{Z}_{2}\right) \rightarrow H^{8}\left(G_{2}\left(\mathbb{R}^{6}\right) ; \mathbb{Z}_{2}\right)$ is zero. Hence, $w_{8}\left(G_{2}\left(\mathbb{R}^{6}\right)\right)$ is not in the image of $H^{6}\left(G_{2}\left(\mathbb{R}^{6}\right) ; \mathbb{Z}\right)$ under the homomorphism $s q^{2}$. Our lemma immediately follows from the following criterion [3]: $M^{8}$ admits a weakly almost complex structure iff $\delta w_{2}(M)=0$ and $w_{8}(M) \in s q^{a} 2 H^{6}(M ; \mathbb{Z})$.

Lemma 2.2. If $G_{k}\left(\mathbb{R}^{n}\right)$ is weakly almost complex, then so are $G_{k-1}\left(\mathbb{R}^{n-2}\right)$ and $G_{k}\left(\mathbb{R}^{n-2}\right)$.

Proof. Let us consider the maps

$$
G_{k-1}\left(\mathbb{R}^{n-2}\right) \stackrel{i}{\rightarrow} G_{k-1}\left(\mathbb{R}^{n-1}\right) \stackrel{j}{\rightarrow} G_{k}\left(\mathbb{R}^{n}\right)
$$


where $i$ regards a $V$ in $\mathbb{R}^{n-2}$ as a $V$ in $\mathbb{R}^{n-1}$ and $j$ sends a $V$ to $V \oplus \mathbb{R}$. It is easy to see that

$$
\begin{aligned}
i^{*}\left(\gamma_{n-1, k-1}\right) & \cong \gamma_{n-2, k-1}, & i^{*}\left(\beta_{n-1, k-1}\right) & \cong \beta_{n-2, k-1} \oplus \varepsilon \\
j^{*}\left(\gamma_{n, k}\right) & \cong \gamma_{n-1, k-1} \oplus \varepsilon, & j^{*}\left(\beta_{n, k}\right) & \cong \beta_{n-1, k-1} .
\end{aligned}
$$

So we have

$$
\begin{aligned}
(j \circ i)^{*} \tau G_{k}\left(\mathbb{R}^{n}\right) & \cong i^{*} \circ j^{*}\left(\gamma_{n, k} \otimes \beta_{n, k}\right) \\
& \cong i^{*}\left(\gamma_{n-1, k-1} \oplus \varepsilon\right) \otimes i^{*}\left(\beta_{n-1, k-1}\right) \\
& \cong\left(\gamma_{n-2, k-1} \oplus \varepsilon\right) \otimes\left(\beta_{n-2, k-1} \oplus \varepsilon\right) \\
& \cong \gamma_{n-2, k-1} \otimes \beta_{n-2, k-1} \oplus \gamma_{n-2, k-1} \oplus \beta_{n-2, k-1} \oplus \varepsilon \\
& \cong \tau G_{k-1}\left(\mathbb{R}^{n-2}\right) \oplus(n-1) \varepsilon .
\end{aligned}
$$

So the conclusion for $G_{k-1}\left(\mathbb{R}^{n-2}\right)$ is true.

Let us consider the maps

$$
G_{k}\left(\mathbb{R}^{n-2}\right) \stackrel{i_{1}}{\rightarrow} G_{k}\left(\mathbb{R}^{n-1}\right) \stackrel{i_{2}}{\rightarrow} G_{k}\left(\mathbb{R}^{n}\right)
$$

By (2.3), we obtain

$$
\begin{aligned}
\left(i_{2} \circ i_{1}\right)^{*} \tau G_{k}\left(\mathbb{R}^{n}\right) & \cong i_{1}^{*} \circ i_{2}^{*}\left(\gamma_{n, k} \otimes \beta_{n, k}\right) \cong i_{1}^{*}\left(\gamma_{n-1, k}\right) \otimes\left(i_{1}^{*}\left(\beta_{n-1, k}\right) \oplus \varepsilon\right) \\
& \cong \gamma_{n-2, k} \otimes\left(\beta_{n-2, k} \oplus \varepsilon \oplus \varepsilon\right) \cong \tau G_{k}\left(\mathbb{R}^{n-2}\right) \oplus 2 \gamma_{n-2, k}
\end{aligned}
$$

By (1.6), $2 \gamma_{n-2, k}$ is in the image of $r: K\left(G_{k}\left(\mathbb{R}^{n-2}\right)\right) \rightarrow K O\left(G_{k}\left(\mathbb{R}^{n-2}\right)\right)$. This completes the proof.

Proof of Theorem 1.1. The statement that $G_{2}\left(\mathbb{R}^{4}\right)$ and $G_{3}\left(\mathbb{R}^{6}\right)$ are weakly almost complex was obtained in [6].

We note that $G_{2}\left(\mathbb{R}^{2 n+1}\right)$ is not weakly almost complex, since it is not orientable. The "only if" part of the theorem may be shown by using this fact, Lemma 2.1, and Lemma 2.2 repeatedly.

Remark. Borel and Hirzebruch [2, p. 526] proved that $G_{2}\left(\mathbb{R}^{n}\right)$ is not almost complex if $n \geq 5$. We extend their results.

\section{THE ORIENTED GRASSMANNIANS}

Proof of Theorem 1.2. If $n$ is odd, $3 \leq k \leq n / 2$, then $\widetilde{G}_{k}\left(\mathbb{R}^{n}\right)$ is not weakly almost complex. The reason is that $w_{3}\left(\widetilde{G}_{k}\left(\mathbb{R}^{n}\right)\right) \neq 0[6]$.

By Lemma 2.1, $G_{2}\left(\mathbb{R}^{6}\right)$ is not weakly almost complex. But $\tau G_{2}\left(\mathbb{R}^{6}\right) \oplus\left(\gamma_{6,2} \otimes\right.$ $\gamma_{6,2} \cong 6 \gamma_{6,2}$. So we see that the element $\gamma_{6,2} \otimes \gamma_{6,2}$ is not in the image of $r: K\left(G_{2}\left(\mathbb{R}^{6}\right)\right) \rightarrow K O\left(G_{2}\left(\mathbb{R}^{6}\right)\right)$.

Let $\xi$ denote the line bundle whose $w_{1}(\xi)$ equals $w_{1}\left(\gamma_{6,2}\right)$. Then $\xi \oplus \gamma_{6,2}$ is an orientable 3-plane bundle with

$$
\left(\xi \oplus \gamma_{6,2}\right) \otimes\left(\xi \oplus \gamma_{6,2}\right) \cong \gamma_{6,2} \otimes \gamma_{6,2} \oplus 2 \gamma_{6,2} \otimes \xi \oplus \varepsilon .
$$

Then we have

$$
\left(\xi \oplus \gamma_{6,2}\right)^{2} \oplus \varepsilon \notin \operatorname{Im} r .
$$

Now let $n$ be even, $k \geq 3$, and $n-k \geq 8=\operatorname{dim} G_{2}\left(\mathbb{R}^{6}\right)$. Since $\widetilde{G}_{k}\left(\mathbb{R}^{n}\right)$ is $(n-k)$-universal for orientable $k$-plane bundles, there exists a map $f: G_{2}\left(\mathbb{R}^{6}\right) \rightarrow$ 
$\widetilde{G}_{k}\left(\mathbb{R}^{n}\right)$ such that $f^{*}\left(\tilde{\gamma}_{n, k}\right) \cong \xi \oplus \gamma_{6,2} \oplus m \varepsilon$, where $m=k-3$. We have

$$
\begin{gathered}
f^{*}\left(\tilde{\gamma}_{n, k} \otimes \tilde{\gamma}_{n, k}\right) \cong\left(\xi \oplus \gamma_{6,2}\right)^{2} \oplus m^{2} \varepsilon \oplus 2 m\left(\xi \oplus \gamma_{6,2}\right), \\
f^{*} \tau \widetilde{G}_{k}\left(\mathbb{R}^{n}\right) \oplus\left(\xi \oplus \gamma_{6,2}\right)^{2} \oplus m^{2} \varepsilon \oplus 2 m\left(\xi \oplus \gamma_{6,2}\right) \cong n f^{*}\left(\tilde{\gamma}_{n, k}\right) .
\end{gathered}
$$

Using (3.1), (1.6), and the fact that $n$ is even, we see that $\widetilde{G}_{k}\left(\mathbb{R}^{n}\right)$ is not weakly almost complex. This completes the proof of the theorem.

\section{ACKNOWLEDGMENTS}

This work was done when the author was at the Max-Planck-Institut für Mathematik in Bonn. I thank Professors F. Hirzebruch and M. Kreck for their hospitality.

\section{REFERENCES}

1. A. Borel and J.-P. Serre, Groupes de Lie et puissances reduites de Steenrod, Amer. J. Math. 75 (1953), 409-448.

2. A. Borel and F. Hirzebruch, Characteristic classes and homogeneous spaces. I, Amer. J. Math. 80 (1958), 458-535.

3. T. Heaps, Almost complex structures on eight and ten-dimensional manifolds, Topology 9 (1970), 111-119.

4. K. Y. Lam, A formula for the tangent bundle of flag manifolds and related manifolds, Trans. Amer. Math. Soc. 213 (1975), 305-314.

5. J. W. Milnor and J. D. Stasheff, Characteristic classes, Ann. of Math. Stud., vol. 76, Princeton Univ. Press, Princeton, NJ, 1974.

6. P. Sankaran, Nonexistence of almost complex structures on Grassmann manifolds, Proc. Amer. Math. Soc. 113 (1991), 297-302.

Department of Mathematics, Graduate School, Academica Sinica, Beijing 100039, People's Republic of China

Max-Planck-Institut für Mathematik, Gottfried-Claren-Strasse 26, D-5300 Bonn 3, GERMANY 